\title{
La ponctuation dans les productions écrites en français des étudiants au Cameroun
}

\author{
Jacques ZRA \\ Faculté des Arts, Lettres et Sciences Humaines, Université de Ngaoundéré - Cameroun
}

Reçu le 6 décembre 2019 | Acceptéle 31 décembre 2019

\begin{abstract}
RÉSUMÉ. Cet article tente d'étudier l'usage des signes de ponctuation par les étudiants dans une université au Cameroun. Il s'appuie sur les copies d'examen recueillies auprès des enseignants. Les erreurs y sont repérées et classées selon leur nature. Elles sont ensuite analysées selon les règles de grammaire. Les résultats révèlent plusieurs types d'erreurs dont l'absence, la confusion et la présence indue de la ponctuation. Au final, une proposition didactique est suggérée pour résoudre le problème observé. Cette étude contribue donc à attirer l'attention des acteurs éducatifs afin qu'ils prennent des mesures dès la base pour juguler les difficultés afférentes à l'usage de la ponctuation.
\end{abstract}

Mots-clés : étudiants, français, productions écrites, signes de ponctuation..

\begin{abstract}
This article attempts to study the use of punctuation by students in one university in Cameroun. It relies on examination copies collected from lecturers and analyzed in order to identify and classify the various mistakes. So, these mistakes are analyzed according to grammar rules. The findings identify particularly the lack of punctuation, confusion and undue presence. Finally, a didactic proposal is suggested to try to solve this problem. This study therefore contributes to attract the attention of those in charge of education so that they take measures to tackle this problem.
\end{abstract}

Keywords: French, punctuation marks, students, written productions.

自 auteur correspondant : zratroustra@gmail.com

Pour citer cet article (Style APA) : Zra, J. (2019). La ponctuation dans les productions écrites en français des étudiants au Cameroun. Francisola: Revue Indonésienne de la langue et la littérature françaises, 4(2), 128-137. doi: 10.17509/francisola.v4i2.24203.

\section{INTRODUCTION}

Parmi les langues qui bénéficient d'un système d'écriture bien normé, la langue française fait partie de celles qui présentent un système graphique très complexe. De Closets (2009) constate que, pour un petit écolier français, l'acquisition de la bonne orthographe prend considérablement plus de temps que pour les écoliers espagnols ou italiens. Sans doute, puisqu'en français, un seul graphème peut représenter deux sons différents. De plus, certains sons n'ont pas d'équivalence graphique alors que ce qui ne s'entend pas sera écrit. Par ailleurs, la ponctuation, un autre élément du système graphique du français, objet de ce travail, ne jouit pas d'une grande facilité d'usage chez les apprenants de cette langue. Il est souvent admis que la ponctuation marquerait des pauses de la langue parlée. Cette hypothèse n'est pas toujours évidente pour le cas du français (Tarnaoui et Hassan, 2018, David et vaudrey-Luigi, 2014). Certes, chaque signe de ponctuation est indiqué par un arrêt, mais tous les arrêts ne correspondent pas toujours à un signe de ponctuation. Par conséquent, on ne peut pas se fier à la respiration pour ponctuer un texte en français. La maîtrise de l'ensemble du système graphique de ladite langue doit être un bien recherché via un enseignement rigoureux afin d'éviter diverses erreurs décrites par des chercheurs à travers des copies d'apprenants (Téguia et Mohamadou, 2015; Benatia Hadj, 2015; 
Belala, 2013 ; etc). La ponctuation française, faut-il rappeler, est fondamentalement en congruence avec syntaxe et sémantique. Et, parmi les différents signes qui la constitue, la virgule semble être celui qui emmerderait beaucoup plus. Et Cavanna de dire : «cette chichiteuse, cette emmerdeuse... on la balancerait volontiers par-dessus bord, elle, ces virgules qu'on ne sait où placer $[\ldots]$ Le point, oui à la rigueur ...» (1994, p.1). Pourtant, c'est un aspect essentiel de la langue qui est négligé, et qu'il faut sérieusement prendre en compte dans le système éducatif pour éviter cet " emmerdement», observé ici dans les copies des étudiants de master, option sciences du langage, puisqu'il s'agit d'eux. Le choix de ce niveau tient au fait que, dans l'imaginaire collectif, les étudiants de langue ont assimilé l'ensemble de paradigmes et de règles qui fondent la grammaire. Loin s'en faut. Le regard jeté sur leurs copies montre que la ponctuation n'est pas leur chose la mieux partagée : absence, confusion, présence indue des signes de ponctuation. En réaction à ce genre de situation, Jaffré (2014) récusait les outils pédagogiques existant en la matière qui ne laissent transmettre que de façon sommaire et partielle les savoirs. Comme le note l'auteur, le cours sur la ponctuation, dans nos écoles, se passe comme s'il était suffisant de les énumérer en signes pausaux ou mélodiques. Alors que les apprenants seront appelés à employer régulièrement les virgules dans les textes ou dans des phrases contenant des structures énumératives ou des compléments déplacés en tête de phrase, à distinguer par exemple la subordonnée relative de la déterminative sans que l'enseignant n'ait tenu un quelconque discours sur ce signe, seulement évoqué. Dans le même ordre d'idée, Besonnat affirme: «de manière massive, la ponctuation jusqu'à ce jour n'a guère fait l'objet d'enseignement au sens strict. Elle s'apprend plutôt incidemment, relève de l'épilinguistique plutôt que du métalinguistique, c'est-à-dire qu'elle est rarement l'objet d'explication » (1991, p.9). Pourtant, il existe une logique d'usage de la ponctuation que l'on gagnerait à cerner pour éviter d'erreurs.

Dans cet article, nous nous concentrerons sur les erreurs de ponctuation contenues dans les copies desdits étudiants et $\mathrm{y}$ proposer une méthode adéquate pour l'apprentissage de cet élément du système graphique du français. Comme nous venons de le dire, les copies que nous avons observées frappent par des signes employés à des endroits non appropriés; d'autres, par ailleurs, sont omis ou confondus abusivement. Ce qui engendre généralement une ambiguïté pouvant entrainer une gêne pour la compréhension du texte (IRHT, 2016). Comme l'a dit Drillon, une «virgule soit mal placée, et c'est une frontière qui se déménage » (1991, p.55). Bien ponctuer le texte consiste, avant toute chose opération, à ordonner sa pensée. À l'inverse, faute de maîtrise, le scripteur ne produira pas de phrases précises, comme observées dans le texte analysé dans ce travail. Les questions suivantes guideront nos analyses : comment les signes de ponctuation y sont-ils employés? Quels sont les types d'erreurs commises? Quelle (s) solution (s) didactique (s) à envisager ?

\section{MÉTHODE}

Le corpus du travail est constitué des copies d'examen des étudiants de master I, option sciences du langage, recueillies, dans le cadre d'une recherche antérieure (Zra, 2014), auprès des enseignants du département de français de l'Université de Ngaoundéré au Caméroun. Il s'agit au total de 57 copies, anonymes.

Après la collecte, la seconde tâche consiste à les dépouiller en profondeur, afin de relever les différentes occurrences d'irrégularités notées. Pour les règles d'usage prises en compte, nous nous référons principalement aux grammaires de Riegel, Pellat et Rioul (2009), Grevisse et Gosse (2008) mais aussi à Doppagne (1998) et Catach (1994), qui enseignent les fonctions de base de la ponctuation française ou la logique ponctuative. Il nous est apparu que ces derniers fournissent d'ample informations pour pouvoir nous permettre d'examiner les 
signes de ponctuation dans les copies qui constituent le corpus de l'étude. En dernier ressort, c'est l'analyse. Ici, nous focalisons l'attention sur les types d'erreurs que nous classons en fonction de leurs catégories : omission, confusion et présence indue.

Compte tenu de l'espace qui nous est imparti, nous ne pouvons pas relever toutes les erreurs de signes de ponctuation commises par ces étudiants dans l'ensemble $\mathrm{du}$ corpus. C'est pourquoi, après avoir considéré que quelques cas, nous fournirons, pour chaque catégorie, des statistiques qui récapitulent la fréquence des erreurs répertoriées dans toutes les copiées analysées. Cela dit, l'étude se veut autant quantitative que qualitative dans la mesure où nous établissons la fréquence des différents types d'erreurs et examinons aussi leurs différentes catégories grammaticales. Nous commençons par le cas d'absence.

\section{RÉSULTATS ET DISCUSSION}

\subsection{Types d'erreurs}

\subsubsection{Omission}

L'omission désigne l'absence de signes de ponctuation à des endroits où les règles de grammaire les recommandent. Cette absence requise par la syntaxe ou par le sens de la phrase influe sur le message que prétend véhiculer l'énonciateur. Elle peut être due soit à l'ignorance de règles de l'écrit, soit à une erreur (Zra, 2014). Et l'erreur, considérée dans la correction comme étant la non maîtrise du système linguistique, est un obstacle à la communication. À première vue, on peut remarquer dans lesdites productions que les signes sont généralement omis après les organisateurs textuels, devant les conjonctions de coordination, entre les mots coordonnés sans conjonctions, devant les propositions explicatives, après les compléments circonstanciels placés en tête de phrases, devant le verbe dont le sujet est éloigné, etc. Nous retenons ici quelques exemples en guise d'illustration.

- Selon les données avancées par l'organisation de la francophonie (OIF)_l'Afrique compte 96 millions de locuteurs sur les 220 millions locuteurs francophones dans le monde. (1)

- À ce moment, la langue française était une langue de prestige, elle était une langue qu'on a surnommée "ne dites pas mais dites plutôt » _ car l'administration coloniale avait imposé une censure très grave pour la personne qui tentera de l'écorcher. (2)

- Les langues étrangères_qui sont issues de la colonisation_ont pris le dessus sur les langues nationales. (3)

- En guise d'illustration nous avons dans l'œuvre de la sénégalaise Nafissatou Niang Diallo intitulée Awa la petite marchande les termes empruntés à la langue pular. (4)

- Le français a connu de nombreuses variations dans le monde où il est parlé (Afrique noire francophone, Europe, Amérique) _ plusieurs facteurs expliquent cette variation (5)

Lorsque nous considérons l'ensemble des copies étudiées, il en ressort que la virgule est de loin le signe qui fait l'objet de plus d'omission. À l'issue du dépouillement, on y enregistre 448 virgules et 217 points qui ont été omis. Dans l'exemple en (1), l'absence d'une virgule se remarque après un organisateur textuel. En effet, lorsque ce dernier n'est pas employé à sa place canonique dans la phrase, il doit être apposé par une virgule (Guénola, 2011). Les organisateurs textuels (adverbes, compléments de phrase ou de nom) sont déplaçables, c'est-à-dire qu'on peut leur changer de place dans la structure phrastique, pourvu qu'ils soient indiqués par une virgule juste après eux. En (2), par contre, l'absence de la virgule est constatée devant la conjonction de coordination «car » qui introduit la proposition coordonnée. Rappelons que la virgule convient toujours devant les conjonctions de coordination autre $n i$, et, ou lorsque les éléments qu'ils introduisent comportent plusieurs propositions. C'est pourquoi, dans cette phrase, elle s'impose devant «car» qui introduit deux propositions : une cordonnée et une relative. Les conjonctions de 
coordination car et mais sont les plus fréquemment concernées (Drillon, 1991 ; Grevisse et Gosse, 2008). Dans l'extrait (3), nous avons un exemple plus complexe que les précédents. Ici, l'omission de la virgule s'observe devant le pronom relatif qui. Certes, l'auteur a la possibilité d'utiliser ou d'omettre cette virgule devant qui, mais cela ne se fait pas de façon hasardeuse. Tout dépend du message à véhiculer. Ici par exemple (en 3), l'absence de la virgule nous donne à croire qu'au Cameroun, toutes les langues étrangères, qui sont issues de la colonisation, ont pris le dessus sur les langues nationales. Ce qui n'est pas vrai. Cela est valable seulement pour le français et l'anglais qui sont des langues officielles du pays. L'allemand, fruit aussi de la colonisation, n'est pas, me semble-t-il, audessus des langues nationales. D'où le problème de véracité de cette phrase. Mais si on plaçait une virgule devant qui et après la fin de la proposition qu'il a introduite, on serait en train de donner juste une indication supplémentaire sur l'origine de ces langues ; par conséquent les éléments intercalés entre deux virgules sont effaçables. Ils sont dits « non essentiels » pour la compréhension du texte et peuvent être supprimés sans incidence sémantique (Chevalier et al. 1964). Alors, ils sont là à titre explicatif et ne détermineraient pas toutes les langues étrangères existantes au Cameroun.

En ce qui concerne les points manquants dans le corpus, ils sont aussi flagrants que les virgules. Pour les remarquer, on n'a pas besoin d'être spécialiste d'un grand effort. L'emploi de point dans un texte se coïncide généralement avec l'observation de pause à l'oral. D'ailleurs, c'est justement ce type de signe qui ont conduit certains auteurs à définir la ponctuation comme la matérialisation des pauses à l'écrit. Mais Thimonnier (1974) a su trancher la question lorsqu'il dit que lors de la lecture à haute voix, chaque signe de ponctuation est marqué par un arrêt, mais que tous les arrêts ne correspondent pas nécessairement à un signe de ponctuation. Si on doit se contenter de la pause pour ponctuer un texte, on ne n'aura au final un produit satisfaisant.

Ici, nous ne donnerons qu'un exemple. L'exemple (5) de nos extraits montre visiblement le manque de ponctuation forte. Nous avons deux phrases dont les idées sont indépendantes, mais elles sont mises en contiguïté, alors qu'une relation de cause à effet demande à être établie avec force entre «Le français a connu de nombreuses variations dans le monde où il est parlé (Afrique noire francophone, Europe, Amérique)» et "plusieurs facteurs expliquent cette variation ». La première idée, qui parle de la variation de la langue, s'arrête à «Amérique» et la seconde, par «plusieurs » et s'arrête à «variation ». Bref, les signes de ponctuation omis dans notre corpus sont nombreux. Le tableau, ci-après, présente une vue d'ensemble de tous ces signes.

Tableau 1. Récapitulation des signes omis

\begin{tabular}{ccccccc}
\hline $\begin{array}{c}\text { Signes } \\
\text { omis }\end{array}$ & Virgules & Points & Point-virgule & Deux-points & Guillemets & Totaux \\
\hline Effectifs & 448 & 217 & 53 & 39 & 37 & 778 \\
Taux $(\%)$ & 57,58 & 27,89 & 6,81 & 5,01 & 4,75 & 100 \\
\hline
\end{tabular}

\subsubsection{Substitution}

La substitution désigne la confusion d'un signe de ponctuation par un autre signe. En effet, on remarque dans le corpus un usage concurrentiel voire ambivalent de certains signes de ponctuation. Les signes qui y sont les plus confondus concernent la virgule, le point, le point-virgule, les deux points. Au total, il en sort environ 180 cas de substitution. La tendance de substitution la plus notoire s'observe entre la virgule et le point. Celle-là est soit placée à l'endroit du celui-ci, soit celui-ci est placé à la place de celle-là. Pourtant, en substance, ces deux signes sont très distincts. En règle générale, le point délimite des unités plus ou moins 
autonomes, tandis que la virgule sépare des unités textuelles perçues comme cohésives, distinctes et non autonomes.

- $\quad$ Retenons, ici, cinq exemples pour illustrer ce cas d'usage des signes ponctuation.

- Nous finissons par l'Europe L ici $^{\prime}$ nous nous intéressons au Canada (6)

- Les langues maternelles sont léguées au second plan dans plusieurs d'Afrique _leur utilisation régresse de plus en plus au Cameroun. (7)

- Le phénomène de contact et de cultures sur le sol camerounais met la pratique du français dans une insécurité._Car aujourd'hui le vocabulaire français est métissé. (8)

- En outre, les langues nationales sont en situation d'infériorité par rapport au français._Parce qu'ils les maintiennent toujours en domination (9)

- Il y a plusieurs facteurs qui expliquent la variation du français dans le monde; nous allons essayer de les expliquer. (10).

Les phrases ci-dessus nous permet de montrer que certains signes de ponctuation ne sont pas employés aux endroits qui conviennent. On remarque certaines unités sont fortement détachées les unes des autres alors sens et syntaxe les unifient. C'est notamment le cas de l'exemple (9). La conjonction "parce que» est placé en tête d'une phrase comportant qu'un seul verbe conjugué. Pourtant, pour reprendre Spengler (1980), «parce que» est un marqueur d'interactivité argumentatif du type justificatif-explicatif. Il est une conjonction et introduit généralement une subordination causale. Celle-ci [subordination], comme sa fonction l'indique déjà clairement, veut dire dans une situation de dépendance à l'autre (la principale), c'est-à-dire qu'elle n'a d'existence que par rapport à la principale. On note la même erreur en (8) où la proposition principale et la coordonnée sont disjointes par la ponctuation forte. En règle générale, les conjonctions car, parce que s'utilisent au niveau interprépositionnel et non, comme c'est le cas en (8) et (9) devant un point de phrase.

Par ailleurs, on remarque des phrases juxtaposées malgré leur complétude sémantique. Ici, les points sont employés à la place des virgules. L'exemple (6) et (7) illustrent ce type d'usage. Les termes « Nous finissons par l'Europe» en (6) sont autonomes sur le plan sémantique et sur le plan syntaxique. La suite en est également. Donc les deux séquences sont indépendantes. Il n'y a pas de raisons valables à les juxtaposées à l'aide d'une virgule. Entre ces deux segments, c'est plutôt un signe de ponctuation fort qui s'impose: le point. Il faut placer un point après le groupe nominal qui «l'Europe ». Il y a une pause forte suggérée non seulement par la diction, mais encore les idées évoquées sont distinctes et nécessiteraient qu'on les donne à voir séparément. Ici, la notion de "phrase» est importante, sinon centrale pour suggérer le signe de ponctuation à utiliser. C'est en fonction de la syntaxe et du sens qu' on opère le signe à utiliser dans ce texte. Alors, même s'il y a un lien de sens entre les phrases cidessus, il s'agirait pour l'auteur d'apporter davantage des précisions dans le raisonnement. Ces exemples laissent aussi montrer visiblement des phrases emboitées les unes dans les autres, « comme le ferait un agencement de parenthèses.

En (10), nous avons un exemple avec le point-virgule. Faut-il rappeler ici quelques règles d'usage de point-virgule? Combinaison de deux signes (virgule et point), le point-virgule est le signe intermédiaire, qui jouerait leurs fonctions synthétiques des deux. Doppagne définit le point-virgule comme un signe qui « tient à la fois du point et de la virgule, mais ne doit pas être compris comme une addition des deux éléments qui le composent : il vaut moins que le point et plus que la virgule» $(1998: 22)$. À ce titre, il marque le caractère indépendant des propositions juxtaposées. Il permet de distinguer les propositions d'une certaine longueur, déjà ponctuées elles-mêmes de virgules obligatoires. Lorsqu'on observe 
l'extrait (10), dans lequel ce signe est employé, on peut se rendre compte, rapidement, de son caractère inadéquat pour peu que l'on se rappelle de la règle que nous évoquions. Encore une fois, il y a deux phrases indépendantes dans cet extrait: « Il y a plusieurs facteurs qui expliquent la variation du français dans le monde » et «Nous allons essayer de les expliquer ». C'est encore deux phrases syntaxiquement et sémantiquement autonomes dont le signe à insérer entre elles est le point de phrase.

Tableau 2. Récapitulation des signes substitués

\begin{tabular}{cccccc}
\hline $\begin{array}{c}\text { Signes } \\
\text { confondus }\end{array}$ & Virgules/points & Points/virgules & $\begin{array}{c}\text { Points- } \\
\text { virgules }\end{array}$ & $\begin{array}{c}\text { Points } \\
\text { d'interrogation/points }\end{array}$ & Totaux \\
\hline $\begin{array}{c}\text { Effectifs } \\
\text { Taux }(\%)\end{array}$ & 76 & 54 & 46 & 5 & 180 \\
\end{tabular}

\subsubsection{Présence indue}

Outre les phénomènes d'absence et de substitution présentées ci-dessus, une autre catégorie repérée, c'est la présence indue de ces signes de ponctuation. La présence indue fait allusion à l'emploi inattendue d'un signe de ponctuation à un endroit donné dans la phrase. Au même titre que les autres catégories, le statut de faute se révèle aussi à une présence non conforme. De ce fait, c'est l'une des règles d'écriture qu'un scripteur doit respecter dans l'usage de la langue, afin que le message soit transmis avec fidélité et pureté. Dans le cas échéant, l'énoncé est paralysé, étant donné que sa présence n'est pas anodine. Nous voudrions parcourir quelques exemples de présence inattendue de ces signes que nous avons pu relever dans les copies étudiées.

1. Le contact des langues $\_$est l'un des facteurs qui explique la variation du français dans le monde (11)

2. Le diatopisme du français est perçu au niveau, phonétique, et sémantique (12)

3. Ils pensent, rêvent $t_{\llcorner}$et s'expriment dans leurs langues maternelles (13)

4. La langue française dans la pratique linguistique quotidienne des camerounais est malaisée. Parce que à côté d'elle se trouve des langues locales qui l'influencent. (14)

5. Dans ce cas, il emprunte les mots dans les langues environnantes, notamment les langues maternelles, anglais, pidgin .....Et on aboutit à la dérive du français par rapport à la norme (15)

L'examen de ces extraits présente trois cas de présence indue des signes de ponctuation. Commençons par l'emploi de la virgule entre le verbe et son sujet (11). En français moderne, la ponctuation n'apparaît pas entre le groupe verbal et son sujet. La présence de la virgule entre le sujet «le contact des langues » et le verbe « être » est une virgule interdite. Spengler dit que les règles de la langue française n'autorisent pas la séparation des constituants syntaxiques fondamentaux de la phrase par un signe de ponctuation; ils sont considérés comme relevant de la syntaxe stricto sensu, c'est-àdire au sens strict. Pour Milner, le lien qui existe entre le sujet et son verbe constituent un domaine. Par domaine, on entendra à la suite de l'auteur, le lien structuré par des lois homogènes, qui ne permet pas la séparation du verbe et son complément par n'importe quel signe de ponctuation. Si on observe l'exemple en (14) en outre, la présence du point n'est pas justifiable. Entre les deux fragments, il $y$ a une relation fondamentalement hypotaxique qui se manifeste grâce au subordonnant "parce que ». Par conséquent, ils ne peuvent pas être isolés par un point de phrase dans l'exemple.

Les extraits (12) et (13) illustrent un autre cas. Ici, nous remarquons l'emploi de la virgule devant « et ». A priori, on s'attend à ce que la conjonction de coordination « et » ne puisse être précéder de la virgule dans ces extraits. Citant Grevisse (1969), Lambert (2006) note qu'on ne sépare pas par la virgule 
les éléments coordonnés par et, ou, ni. Mais il mentionne, toujours en citant Grevisse, que dans certains cas, les conjonctions et, ou, ni sont précédées de la virgule. Tout dépend en réalité du contexte. Il s'impose donc ici de préciser quelques cas où l'usage de la virgule est légitime devant et afin de voir si elle est à sa place dans les extraits ci-dessus. Il y a plusieurs cas possibles.

On utilise la virgule devant et pour permettre de distinguer des niveaux de parenthésage, lorsque et est répété (1). La séparation introduite devant et permet de faire remonter le niveau coordinatif aux ensembles placés avant et déjà reliés par et » (2). Un autre cas de figure susceptible de motiver la présence d'une virgule devant et correspond à un décalage syntaxique entre les éléments conjoints. De même, les grammaires citées suggèrent que la virgule apparaît devant et quand le sujet change. (Lambert, 2006 : 208-209).

Grosso modo, si l'emploi de la virgule devant et peut être justifié, les cas repérés dans notre corpus ne sont pas conformes aux prescriptions grammaticales ou linguistiques. La virgule est employée devant et plutôt dans l'énumération pour introduire le dernier élément. L'énumération n'autorise jamais l'emploi de la virgule devant et.

L'exemple (15) illustre un cas d'usage de point utilisé en surnuméraire aux points de suspension. Certes, les fonctions des petits points, comme ils sont aussi appelés, sont nombreuses, mais celles qui ont amené les étudiants à l'utiliser dans leur production indiquent l'interruption d'un texte ou la continuité d'une énumération. Mais lorsque les points de suspension se trouvent à la fin d'une phrase, ils jouent le rôle de point de phrase. Il s'agit de la loi d'absorption dont parle Tournier en des termes suivants : « Il existe des signes de ponctuation qui ne peuvent pas apparaître l'un à côté de l'autre, bien comportant des ponctuants et des ponctuances différents : dans tel cas, un seul ponctuant est réalisé et il se charge alors de sa ponctuance propre et des autres ». De fait, à partir du moment où les points de suspension sont employés à la fin de la phrase, ils équivalent au point de phrase ; celui-ci ne doit plus se retrouver plus en situation de co-présence.

Ainsi, la ponctuation est l'élément fondamental de la langue écrite. Sa maîtrise doit être un bien recherché. Notons avec Lemaître (1995) que l'expression écrite est une tâche scolaire. La ponctuation, élément carrefour de cette pratique, devrait témoigner ou caractériser cet apprentissage. Et si les apprenants ont des difficultés à l'utiliser grammaticalement, il est important d'attirer l'attention des acteurs éducatifs.

Tableau 3. Récapitulation des emplois des signes indus

\begin{tabular}{ccccccc}
\hline Signes indus & Virgules & Points & $\begin{array}{c}\text { Points- } \\
\text { virgules }\end{array}$ & Deux-points & $\begin{array}{c}\text { Points de } \\
\text { suspension }\end{array}$ & Totaux \\
\hline Effectifs & 52 & 28 & 6 & 7 & 4 & 97 \\
Taux $(\%)$ & 53,60 & 28,86 & 6,18 & 7,21 & 4,12 & 100 \\
\hline
\end{tabular}

\subsection{Proposition didactique pour l'enseignement de la ponctuation au secondaire}

Comme tenu des types des signes qui présentent les plus grands nombres d'erreurs dans notre corpus, il serait important pour son apprentissage d'adopter la méthode d'ingénierie didactique proposée par Artigues, qui consiste à passer de l'oral à l'écrit. L'objectif des activités proposées est de faire comprendre la différence entre les deux formes de la langue (française). La spécificité de la méthode réside dans l'opposition de la phrase graphique à la «phrase orale» en montrant que ce sont deux types de productions langagières d'une langue, et que les caractéristiques ne sont pas les mêmes. Car, comme l'ont affirmé More et Danon-Boileau : «De l'oral à l'écrit, il y a un monde» (1998: 7). Pour ce faire, nous proposons quelques étapes à suivre, que nous avons à peine remaniées, énoncées par Campana (2002 : 72) :

- Le professeur donne à lire une transcription écrite d'un discours 
oral. Il propose le texte sans aucune marque de ponctuation.

- L'enseignant fait écouter cet extrait oral. Les apprenants entendent alors les pauses et les intonations de l'oral.

- Lors d'une deuxième écoute, la tâche des élèves est de noter par un trait vertical les pauses qu'ils perçoivent. Lors d'une mise en commun, une harmonisation des propositions de segmentation a lieu.

- Lors d'une troisième écoute, les élèves doivent être attentifs à la longueur des pauses et aux différents types d'intonations qui coïncident avec ces pauses : soit montantes, soit descendantes.

- On demande ensuite de recopier l'énoncé transcrit en plaçant la ponctuation et les majuscules qui semblent correspondre aux deux types de pauses que les élèves ont identifiées. Les phrases obtenues ne sont pas acceptables: on doit conclure qu'il n'y a pas de correspondance entre pauses, intonation et ponctuation.

- La dernière étape de la séquence consiste à réécrire la transcription du récit oral de telle sorte qu'elle soit acceptable à l'écrit. Parmi les versions proposées, un texte est choisi et recopié par l'enseignant au tableau. Le maître lit à haute voix le texte en demandant aux de porter leur attention sur les liens entre intonations et pauses. Les élèves doivent avoir conscience que les pauses marquées sont celles de la lecture à haute voix.

En procédant ainsi, on aura attiré l'attention des apprenants de la grande différence entre l'oral et l'écrit. Par conséquent, les élèves retiendraient, nous semble-t-il, l'idée selon laquelle une pause à l'oral n'équivaut pas toujours à un signe de ponctuation. "Sur un plan didactique, il s'agirait de s'affranchir de la traditionnelle référence à l'oral, encore très présente dans les manuels scolaires, pour poser les enjeux, indépendamment des phénomènes suprasegmentaux, en termes sémiotiques, syntaxiques et sémantiques » (Rault, 2014, p.17).

\section{CONCLUSION}

En somme, nous nous sommes proposé d'examen des erreurs des signes de ponctuation commises par les étudiants dans ce travail. L'analyse de ces fautes révèle plusieurs catégories: il manque un signe de ponctuation (omission), un signe est employé à la place d'un autre signe (substitution), et enfin un signe est employé à la place d'un blanc (présence indue). Dans la catégorie d'omission, nous avons repéré 778 cas. En ce qui la substitution, il en ressort environ 180 signes confondus, créant ainsi une rupture entre certains segments qui sont liés sur les plans syntaxique et sémantique. D'autres, juxtaposés malgré leurs caractères indépendants. La dernière catégorie de fautes, commises par ces apprenants, est la présence inattendue de certains signes. Nous en avons dénombré 97 signes, utilisés alors qu'ils ne sont pas requis ni par la syntaxe ni par le sens des phrases concernées. Lorsque nous portons un regard global sur ces résultats, un constat s'impose : la virgule est de loin le signe de ponctuation qui a constitué la plus grande source d'erreurs. Cela est dû à sa nature plus complexe. Dugas (1997) l'a déjà qualifié comme celle qui tient le haut du pavé, parmi les grands mystères de la vie, aux côtés des trous noirs et des humeurs de l'ordinateur. Face à cette situation, l'on s'interroge sur la meilleure méthode didactique à choisir pour remédier le problème. On sait que l'enseignement de la ponctuation dans nos lycées et collèges passe généralement à l'occasion des cours sur les types de phrases. Quand bien même elle est souvent enseignée, mais c'est de façon énumérative, sans moindre discours sur leurs rôles dans des phrases. Il est important, nous semble-t-il, que les enseignants, surtout du secondaire, l'abordent en comparant l'oral et l'écrit. C'est pourquoi, nous suggérons dans ce travail la méthode d'ingénierie didactique d'Artigues qui, de notre point de vue, permet 
de mieux faire comprendre les spécificités de l'un et de l'autre, et par conséquent de la nature indépendante de certains signes de ponctuation par rapport aux pauses à l'oral.

\section{REMERCIEMENTS}

J'ai bénéficié de l'encadrement inégalable de la Pr Léonie Métangmo-Tatou dans mes différents travaux de recherche, notamment dans le domaine de la ponctuation française. Aujourd'hui, si je me permets d'en parler, c'est grâce à cette imminente chercheuse, qui n'a jamais ménagé aucun effort à m'encourager dans mes travaux. C'est elle qui a mis à ma disposition les copies qui m'ont servi ici du corpus. Je luis en sais reconnaissant! Je remercie aussi Dr Daouaga Samari Gilbert pour ses conseils et ses remarques dans le cadre de cet article.

\section{RÉFÉRENCES}

Artigues, M. (2002). «Ingénierie didactique : quel rôle dans la recherche didactique aujourd'hui ». Les dossiers des sciences de l'éducation 8, pp. 59-73. https://www.persee.fr/doc/dsedu 1296 -2104 2002 num 811010

Belala, M. H. (2013). L'orthographe dans les productions écrites des élèves de la première année moyenne. Mémoire de master, Université Algérienne démocratique et populaire. https://bu.umc.edu.dz/theses/francais/ BEL1335.pdf

Besonnat, D. (1991). «Enseigner la ..."ponctuation" ? (!). In : Pratiques : linguistique, littérature, didactique, $\mathrm{n}^{\circ}$ 70, pp. 9-45. Repéré à https://www.persee.fr/doc/prati 033823891991 num 7011635

Bogni, T. et Mohamadou, O. (2015). «Pratiques de la dictée en classe de français dans l'enseignement secondaire au Cameroun. Analyse des approches et de la performance d'élèves dans un lycée à Ngaoundéré ». In Brissaud, C. et Mortamet, C. (dir.) La dictée, une pratique sociale emblématique, Glottopol, No 26, pp. 58-68. http://glottopol.univ- rouen.fr/telecharger/numero 26/gpl26 complet.pd

Campanna, M. (2002). Une grammaire pour mieux écrire. CRDP de Créteil.

Catach, N. (1994). La ponctuation. Paris : PUF.

Charef bénatia, H. (2015). Les erreurs d'orthographe dans l'écrit chez les apprenants de la 4eme moyenne CEM de Sirat. Mémoire de master, Université Addel Hamid ibn à Mostaganem. http://e-biblio.univmosta.dz/bitstream/handle/123456789/ 6881/chare

David, J. et Vaudrey-Luigi, S. (2014). «Enseigner la ponctuation». Le français aujourd'hui, Armand Colin, 187 (4). pp. 3-6. https://www.cairn.info/revue-lefrancais-aujourd-hui-2014-4-page3.htm

De Closets, F. (2009). Zéro faute. L'orthographe, une passion française. Éditions Mille et une nuits, Villeneuve-d'Ascq.

Doppagne, A. (1998). La bonne ponctuation, 3eme édition. Collection «entre guillemets », paris-Bruxelles : Ducolot.

Drillon, J. (1991). Traité de ponctuation française. Paris : Gallimard.

Dugas, A. (1997). Le guide de la ponctuation française. Montréal: Les Éditions logiques.

Grevisse, M. et Gosse, A. (2008). Le bon usage : grammaire française, $14 \mathrm{e}$ édition, Bruxelles et Louvain-La-neuveÈdition De Boeck et Ducolot.

Guénola, J. (2011). Enseigner la ponctuation et apprendre la grammaire: le cas de la phrase et de la ponctuation au Cycle II. Thèse de doctorat, Université Toulouse le Mirail. https://tel.archivesouvertes.fr/tel-00620750v1

Jaffré, J.P. (2014). «Postface : à quoi sert la ponctuation », Armand Colin, n ${ }^{\circ} 187$, pp. 129-135. https://www.cairn.info/revue-lefrancais-aujourd-hui-2014-4-page129.htm

Lambert, F. (2006). «Pourquoi met-on une virgule devant et?", Cahier de grammaire, $\mathrm{n}^{\circ}$ 30, pp. 205-218. http://w3.erss.univ- 
tlse2.fr/textes/publications/CDG/30/CG 30-16-Lambert.pdf

Lemaître, B. (1995). "La ponctuation: un savoir enseignable? enseigné ? In : Revue de recherches en éducation, No, pp. 161-195

https://www.persee.fr/doc/spira 0994$\underline{37221995 \text { num } 1511911}$

Rault, J. (2014). La ponctuation: problématiques linguistiques. Le

français aujourd'hui, Armand Colin, 187(4), 9-18. doi:10.3917/lfa.187.0009. https://www.cairn.info/revue-lefrancais-aujourd-hui-2014-4-p-9.htm

Riegel, M., Pellat, J.-C. et Rioul, R. (2009). Grammaire méthodique du français. Paris: Presses Universitaires de France.

Spengler, N. (1980). « Première approche de marque d'interactivité de la ponctuation", cahier de linguistique française, $\quad \mathrm{n}^{\circ} \quad 1, \quad 128-148$. https://clf.unige.ch/files/3714/4111/1969 L07-Spengler_nclf1.pdf
Tarnaoui, M.M. et Hassan, B. (2018). La ponctuation des lycées morocains du FLE. Défauts textuels et dispositifs de rémédiation. Francisola: Revue indonésienne de langue et de littérature, 3 (2), pp. 141-152. http://dx.doi.org/10.17509/francisola.v3 i2.15747

Thimonnier, R. (1974). Code typographique et grammatical. Paris : Hatier.

Tournier, C. (1980). « Histoire des idées sur la ponctuation, des débuts à l'imprimerie à nos jours », langue française 45 , pp. 28-40.

https://www.persee.fr/doc/lfr 002383681980 num 4515261

Zra, J. (2014). La ponctuation dans les productions écrites en français des étudiants de l'Université de Ngaoundéré. Mémoire, Université de Ngaoundéré. Non publié. 